\title{
Theory of surface noise under Coulomb correlations between carriers and surface states
}

\author{
V. A. Kochelap and V. N. Sokolov \\ Department of Theoretical Physics, Institute of Semiconductor Physics, Pr. Nauki 45, Kiev 03028, Ukraine \\ O. M. Bulashenko a) and J. M. Rubí \\ Departament de Física Fonamental, Universitat de Barcelona, Diagonal 647, E-08028 Barcelona, Spain
}

(Received 7 May 2002; accepted 14 August 2002)

\begin{abstract}
We present a theory of the surface noise in a nonhomogeneous conductive channel adjacent to an insulating layer. The theory is based on the Langevin approach which accounts for the microscopic sources of fluctuations originated from trapping-detrapping processes at the interface and intrachannel electron scattering. The general formulas for the fluctuations of the electron concentration, electric field as well as the current-noise spectral density have been derived. We show that due to the self-consistent electrostatic interaction, the current noise originating from different regions of the conductive channel appears to be spatially correlated on the length scale correspondent to the Debye screening length in the channel. The expression for the Hooge parameter for $1 / f$ noise, modified by the presence of Coulomb interactions, has been derived. (C) 2002 American Institute of Physics. [DOI: 10.1063/1.1512698]
\end{abstract}

\section{INTRODUCTION}

IBM Corporation has announced recently ${ }^{1}$ the principal strategy on silicon-on-insulator (SOI) technology as a key platform to manufacture logic integrated circuits for digital and $\mathrm{rf}$ low-power, low-voltage applications. ${ }^{2}$ Downscaling SOI technologies in general raises the $1 / f$ noise level due to the increased role of the semiconductor-insulator interface, which introduces specific noise sources. ${ }^{3}$ This is the socalled surface noise caused by random trapping and detrapping of carriers to and from the surface states of conducting channels. This noise is especially pronounced in the devices operating in the high-field regime, since it is proportional to square of the electric field. ${ }^{4}$

The surface noise causes a great impact on the device performance, as was observed in different scaled-down devices. [See, e.g., recent experimental studies on metaloxide-semiconductor field-effect transistors (MOSFETs), ${ }^{3,5}$ thin-film transistors (TFTs), ${ }^{6}$ polysilicon emitter bipolar junction transistors (BJTs), ${ }^{7}$ and heterostructure field-effect transistors (HFETs) ${ }^{8,9}$ ] In a class of heterostructure materials based on the group-III nitride wide-gap semiconductors, ${ }^{10}$ the fluctuations are caused mainly by the surface noise. ${ }^{9}$ The use of the devices with reduced dimensions implies that the noise modeling for practical design purposes becomes more complex. The demand for improved theoretical descriptions is even more relevant from a device simulation viewpoint: most of the $1 / f$-noise models included in standard simulation tools are too simple, since they ignore the electron correlations due to electrostatic effects and the inhomogeneous nature of the channel transport. These effects may often be significant, as was recently demonstrated. ${ }^{11}$ Therefore, the currently existing theories should be replaced by more accurate physics-based descriptions that include all those effects.

\footnotetext{
${ }^{a)}$ Electronic mail: oleg@ffn.ub.es
}

In this article, we present a theory of the surface noise in a nonhomogeneous conductive channel taking into account the electrostatic effects and transverse electron transport. The theory is based on the Langevin approach, which accounts for the microscopic sources of fluctuations originated from trapping-detrapping processes at the interface and intrachannel electron scattering. The general formulas for the fluctuations of the electron concentration, electric field as well as the current-noise spectral density have been derived. We show that the electrostatic (Coulomb) correlations among the trapped and channel electrons considerably affect the surface noise leading to its suppression.

The necessity of the self-consistent treatment requires an additional discussion. In general, Coulomb correlations appear when the average distance between the traps in the dielectric is less or of the order of the Debye screening length $l_{D}=\left(\varepsilon k_{B} T / q^{2} n\right)^{1 / 2}$, where $\varepsilon$ is the dielectric constant, $T$ is the temperature, $q$ the electron charge, and $n$ the bulk electron concentration. This implies

$$
\pi l_{D}^{2} N_{t} \gtrsim 1,
$$

where $N_{t}$ is the sheet concentration of traps in the dielectric. Therefore, the trap concentration should exceed some characteristic value $N_{t 0} \equiv 1 /\left(\pi l_{D}^{2}\right)$. For a channel of effective thickness $\sim l_{D}$, one may introduce the average sheet electron concentration $N=n l_{D}$. Then, the substitution $n=N / l_{D}$ into the expression for the Debye length gives $l_{D}$ $=\left(\varepsilon k_{B} T / q^{2} N\right)$. Thus, condition (1) for the self-consistent treatment of Coulomb correlations leads to the relation between the sheet concentration of traps and the sheet electron density in the channel:

$$
N_{t} \gtrsim N_{t 0}=\frac{1}{\pi}\left(\frac{q^{2} N}{\varepsilon k_{B} T}\right)^{2} \propto N^{2}
$$


The estimations of the critical values $N_{t 0}$ for different semiconductor materials and channel concentrations will be given below in Sec. VI C.

The article is organized as follows. In Sec. II, we describe the physical model of a nonhomogeneous conducting channel with Coulomb interactions between the trapped and channel electrons. The basic equations are given as well. In Sec. III, the equation for the electric field fluctuations is solved analytically. Then, the spectral density of carrier number fluctuations is calculated with the emphasis on the difference with the results of McWhorter's model: the extra factors associated with Coulomb correlations and transverse electron transport in the channel. In Sec. IV, the current-noise spectral density is calculated and the surface-noise suppression factor is defined. The spatial correlations of the noise arising due to the Coulomb interactions between the trapped and free electrons are considered in Sec. V. Next, in Sec. VI, we discuss the results obtained for two different models: traps uniformly distributed over the layer and in-plane distribution of traps at a certain distance from the interface. The frequency and intensity behavior of the noise spectrum is analyzed. Finally, some additional derivations are presented in the Appendices: the reduction of three-dimensional stochastic equations to a one-dimensional form (Appendix A), and an analysis of the rate constants for trapping and detrapping processes (Appendix B).

\section{MODEL AND BASIC EQUATIONS}

We consider a semiconductor channel with electron conductivity and with lateral dimensions $L_{x} \times L_{z}$ near an adjacent dielectric layer (Fig. 1). The $y$ axis is taken normal to the plane of the interface. External electric field $E_{\|}$is applied along the direction $x$ parallel to the interface. In the transversal direction, the spatial profiles of the electrostatic potential $\varphi(y, t)$ and electron concentration $n(y, t)$ inside the channel $(y>0)$ are strongly nonhomogeneous and they are determined by the surface potential $\varphi_{s}$ and the Fermi level $\varepsilon_{F}$. Below, we use the subscript $s$ for the values taken at the surface $y=0$. The concentration of trapped electrons in the dielectric layer $(y<0)$ is denoted by $n_{k}(y, t)$, where the subindex $k$ indicates different traps levels. The trapping potential is supposed to be of the short range, which is typical for deep levels in the dielectric.

The basic semiclassical transport equations for nondegenerate electrons, including both steady states and fluctuations as well as Langevin noise sources, can be written in a one-dimensional form with respect to the transversal coordinate $y$ (see Appendix A):

$$
\begin{aligned}
& i_{\perp}(y, t)=-\mu E_{\perp} n-D \frac{\partial n}{\partial y}+\delta J_{\perp}(y, t), \\
& \frac{\partial E_{\perp}}{\partial y}=\frac{\rho(y)}{\varepsilon}, \\
& \frac{\partial n(y, t)}{\partial t}+\frac{\partial i_{\perp}}{\partial y}=0,
\end{aligned}
$$

$$
\frac{\partial n_{k}(y, t)}{\partial t}=S_{k} n(0, t)-K_{k} n_{k}+\delta Q_{k}(y, t) .
$$

Here, $i_{\perp}$ is the transverse electron flux, $\delta J_{\perp}$ the corresponding Langevin source usually called diffusion or thermal noise source, $E_{\perp}=-(\partial \varphi / \partial y)$ is the electric field perpendicular to the interface, $\mu$ the electron mobility, $D$ the diffusion coefficient, $\rho(y)$ the channel charge distribution, $S_{k}$ and $K_{k}$ the rate constants for trapping and detrapping processes, respectively (Appendix B), and $\delta Q_{k}$ the trapping noise source. We assume that inside the channel far away from the interface a local charge neutrality and zero transversal electric field are reached. In addition, the conservation of the total charge in the whole system: the channel and the dielectric layer is imposed. Then, the boundary conditions to Eqs. (4) and (5) can be written as

$$
\rho(\infty)=0, \quad E_{\perp}(\infty)=0,
$$

and

$$
i_{\perp, s}=-\int_{y<0} d y \sum_{k}\left(S_{k} n_{s}-K_{k} n_{k}+\delta Q_{k}\right) .
$$

We show in Appendix A that the spectral correlators for the Langevin sources of noise are expressed as

$$
\begin{aligned}
& \left\langle\delta J_{\perp}(y) \delta J_{\perp}\left(y^{\prime}\right)\right\rangle_{\omega}=4(D / A) n(y) \delta\left(y-y^{\prime}\right), \\
& \left\langle\delta Q_{i}(y) \delta Q_{k}\left(y^{\prime}\right)\right\rangle_{\omega}=4\left(K_{k} / A\right) n_{k}(y) \delta_{i k} \delta\left(y-y^{\prime}\right), \\
& \left\langle\delta J_{\perp}(y) \delta Q_{k}\left(y^{\prime}\right)\right\rangle_{\omega}=0,
\end{aligned}
$$

where $n(y)$ and $n_{k}(y)$ are the steady-state concentrations of free and trapped electrons, respectively, and $A=L_{x} L_{z}$ is the effective area of the interface. Each Langevin source $\delta J_{\perp}$, $\delta J_{\|}$, and $\delta Q_{k}$ is supposed to be correlated only with itself. Assuming further for simplicity that the conductive channel is uniform in the $x z$ plane, the electron density flux along the channel can be written as

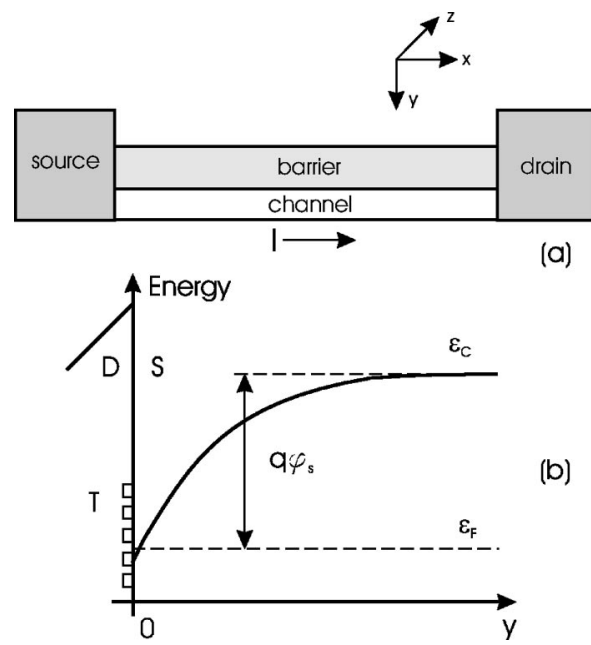

FIG. 1. (a) Schematic view of a heterostructure formed by a semiconductor channel adjacent to an insulating barrier. The current flows from "source" to "drain" along the channel. (b) Cross-sectional band-energy diagram of the heterostructure. Trap states $(T)$ are at the dielectric-semiconductor $(D-S)$ interface. $\varepsilon_{c}$ is the conduction-band edge, $\varepsilon_{F}$ the Fermi level, and $q \varphi_{s}$ the surface band bending. 


$$
i_{\|}(y, t)=-\mu E_{\|} n(y, t)+\delta J_{\|}(y, t),
$$

where $\delta J_{\|}$is the Langevin longitudinal flux whose correlator is similar to that for the transverse random flux: $\left\langle\delta J_{\|}(y) \delta J_{\|}\left(y^{\prime}\right)\right\rangle_{\omega}=4(D / A) n(y) \delta\left(y-y^{\prime}\right)$.

The instantaneous local variables in Eqs. (3)-(8) can be split into steady-state and fluctuating quantities:

$$
\begin{aligned}
& n(y, t)=n(y)+\delta n(y, t), \\
& E_{\perp}(y, t)=E_{\perp}(y)+\delta E_{\perp}(y, t), \\
& i_{\perp}(y, t)=i_{\perp}(y)+\delta i_{\perp}(y, t), \\
& n_{k}(y, t)=n_{k}(y)+\delta n_{k}(y, t),
\end{aligned}
$$

where the fluctuating components are denoted by $\delta$. The stationary problem is strongly nonlinear, however, all the steady-state profiles $n(y), E_{\perp}(y)$, and $n_{k}(y)$ can be found as implicit functions of $y .{ }^{12}$ Our main goal is to solve the fluctuation problem. Substituting Eqs. (11) into Eqs. (3)-(6) and retaining the terms linear in fluctuations, ${ }^{13}$ we have

$$
\begin{aligned}
& \delta i_{\perp}(y, t)=-\mu\left(n \delta E_{\perp}+E_{\perp} \delta n\right)-D \frac{\partial \delta n}{\partial y}+\delta J_{\perp}(y, t), \\
& \frac{\partial \delta E_{\perp}}{\partial y}=\frac{\delta \rho(y)}{\varepsilon}, \\
& \frac{\partial \delta n(y, t)}{\partial t}+\frac{\partial \delta i_{\perp}}{\partial y}=0, \\
& \frac{\partial \delta n_{k}(y, t)}{\partial t}=S_{k} \delta n(0, t)-K_{k} \delta n_{k}+\delta Q_{k}(y, t) .
\end{aligned}
$$

In the next section, we shall obtain the analytical solution to this system of partial differential equations subject to the boundary conditions (7) and (8). This will allow us to evaluate the spectral densities of fluctuations of the electric field and carrier density.

\section{FLUCTUATIONS OF ELECTRIC FIELD AND CARRIER DENSITY}

First, we reduce Eqs. (12)-(14) for the channel variables to a second-order differential equation for the fluctuation of the transverse electric field and find the analytical solution to this equation. Then, the density fluctuations of free and trapped electrons, $\delta n$ and $\delta n_{k}$, which are coupled selfconsistently with $\delta E_{\perp}$, can be obtained directly from Eqs. (13) and (15), respectively. Having found a full solution to the problem described by Eqs. (12)-(15) and Eqs. (7) and (8), we can evaluate the spectral densities for fluctuations of carrier density and electric field that determine the excess noise spectrum.

From Eq. (13) with $\delta \rho=-q \delta n$ and Eq. (14), we find

$$
q \delta i_{\perp}(y, t)-\varepsilon \frac{\partial \delta E_{\perp}(y, t)}{\partial t}=C(t),
$$

where the integration constant $C(t)$ is independent of the coordinate $y$. It has the meaning of the fluctuation of the transverse current, which includes two components: the drift-diffusion and displacement contributions. By assuming that the fluctuation of the total transverse current is zero, we may put $C(t)=0$. Moreover, the characteristic times of the trapping-detrapping processes $S_{k}^{-1}$ and $K_{k}^{-1}$ are usually much greater than the dielectric relaxation time $\tau_{d}$ $=\epsilon /(q \mu n)$. Thus, for the relevant frequency range $\omega \tau_{d} \ll 1$, the displacement current component can be dropped, and then it follows from Eq. (16) that

$$
\delta i_{\perp}=0 .
$$

Using Eq. (17), one can easily find the equation for the Fourier component of the fluctuation of the transverse electric field $\delta E_{\perp}^{\omega}(y)$ in the form

$$
\left[\frac{\partial^{2}}{\partial y^{2}}+\frac{\mu}{D} E_{\perp}(y) \frac{\partial}{\partial y}-\frac{q \mu}{\epsilon D} n(y)\right] \delta E_{\perp}^{\omega}(y)=-\frac{q}{\epsilon D} \delta J_{\perp}^{\omega}(y) .
$$

Here, the second-order differential operator in square brackets may be transformed to a self-adjoint form by the substitution $\mu E_{\perp} / D=-W^{\prime} / W$, where prime stands for the derivative with respect to $y$. Integrating this relation from 0 to $y$, one gets $W(y)=W(0) \exp \left\{\mu\left[\varphi(y)-\varphi_{s}\right] / D\right\}$. In this result, the constant factor $W(0)$ can be taken equal to 1 , since it will be canceled when the expression for $W(y)$ is substituted into the general solution (see below). Now, Eq. (18) becomes

$$
\hat{L} \delta E_{\perp}^{\omega}(y)=-\frac{q}{\varepsilon} \delta s^{\omega}(y),
$$

with

$$
\hat{L}=\frac{\partial}{\partial y}\left[\frac{1}{W(y)} \frac{\partial}{\partial y}\right]-\frac{q \mu}{\epsilon D} \frac{n(y)}{W(y)},
$$

where we have denoted the effective Langevin source $\delta s^{\omega}(y) \equiv \delta J_{\perp}^{\omega}(y) /[D W(y)]$. The general solution to Eq. (19) can be found in an analytical form by making use of the method of finding solutions of stochastic equations developed in our previous papers. ${ }^{14}$ We get

$$
\begin{aligned}
\delta E_{\perp}^{\omega}(y)= & \frac{\delta E_{\perp, s}^{\omega}}{u_{1, s}} u_{1}(y)+u_{1}(y) \int_{0}^{y} u_{2}(\xi) \delta r^{\omega}(\xi) d \xi \\
& +u_{2}(y) \int_{y}^{\infty} u_{1}(\xi) \delta r^{\omega}(\xi) d \xi,
\end{aligned}
$$

where $u_{1,2}(y)$ are two linearly independent solutions of the homogeneous equation corresponding to Eq. (19), and $\delta r^{\omega}(y) \equiv(q / \varepsilon) \delta s^{\omega}(y)$. It is convenient to choose $u_{1}(y)$ $=d E_{\perp} / d y=\rho(y) / \varepsilon$, since it satisfies the equation $\hat{L} \rho(y)$ $=0$. Then, we can write $u_{2}(y)=\varepsilon \rho(y) \int_{0}^{y}\left[W(\xi) / \rho^{2}(\xi)\right] d \xi$, since $W$ is the Wronskian for the functions $u_{1,2}$. To obtain Eq. (21), we have used the conditions: $u_{1}(\infty)=0$ and $u_{2}(0)=0$. The correlation properties of the stochastic source $\delta r^{\omega}(y)$ follow from Eqs. (9):

$$
\left\langle\delta r(y) \delta r^{*}\left(y^{\prime}\right)\right\rangle_{\omega}=\frac{4 q^{2} n(y)}{\varepsilon^{2} A D W^{2}(y)} \delta\left(y-y^{\prime}\right) .
$$

Having found $\delta E_{\perp}^{\omega}(y)$, we can evaluate the electron density fluctuation in the channel through $\delta n^{\omega}(y)=-(\varepsilon / q)$ $\times\left(d \delta E_{\perp}^{\omega} / d y\right)$. This yields 


$$
\begin{aligned}
\delta n^{\omega}(y)= & -\frac{\varepsilon}{q}\left\{\frac{\delta E_{\perp, s}^{\omega}}{u_{1, s}} u_{1}^{\prime}(y)+u_{1}^{\prime}(y) \int_{0}^{y} u_{2}(\xi) \delta r^{\omega}(\xi) d \xi\right. \\
& \left.+u_{2}^{\prime}(y) \int_{y}^{\infty} u_{1}(\xi) \delta r^{\omega}(\xi) d \xi\right\}
\end{aligned}
$$

In particular, at the surface it is given by

$$
\delta n_{s}^{\omega}=-\frac{\varepsilon}{q u_{1, s}}\left\{u_{1, s}^{\prime} \delta E_{\perp, s}^{\omega}+\int_{y>0} u_{1}(y) \delta r^{\omega}(y) d y\right\} .
$$

Introducing the average sheet electron density in the channel $N=\int_{0}^{\infty} n(y) d y$ and its fluctuation $\delta N=\int_{0}^{\infty} \delta n(y) d y$, one can find that $\delta N$ is related to the fluctuations of the electric field at the boundaries

$$
\delta N^{\omega}=\frac{\varepsilon}{q}\left[\delta E_{\perp}^{\omega}(0)-\delta E_{\perp}^{\omega}(\infty)\right] .
$$

A useful consequence of Eq. (25) is the relation between the spectral correlators

$$
\left\langle\delta N \delta N^{*}\right\rangle_{\omega}=\left(\frac{\varepsilon}{q}\right)^{2}\left\langle\delta E_{\perp, s} \delta E_{\perp, s}^{*}\right\rangle_{\omega},
$$

in which we have taken into account the boundary conditions (7). It will be used in the next section in evaluation of the noise spectrum.

The electric field fluctuation $\delta E_{\perp, s}^{\omega}$ is still unknown. To find it, we employ the additional condition: the conservation of the total charge in the conducting channel and dielectric layer

$$
\delta N^{\omega}+\int_{y<0} d y \sum_{k} \delta n_{k}^{\omega}(y)=0 .
$$

The density fluctuations of trapped electrons $\delta n_{k}^{\omega}$ are obtained from Eq. (15) as

$$
\delta n_{k}^{\omega}(y)=\beta_{k}(\omega) S_{k}(y) \delta n_{s}^{\omega}+\beta_{k}(\omega) \delta Q_{k}^{\omega}(y),
$$

where $\beta_{k}(\omega) \equiv\left[K_{k}(y)+i \omega\right]^{-1}$, and the fluctuation $\delta n_{s}^{\omega}$ is given in Eq. (24). Combining Eqs. (24), (25), (27), and (28), we obtain

$$
\delta E_{\perp, s}^{\omega}=-\frac{(q / \varepsilon) \delta N_{\mathrm{td}}^{\omega}-\left(1 / \rho_{s}\right) \eta(\omega) \int_{y>0} \rho(y) \delta r^{\omega}(y) d y}{1-\left(\rho_{s}^{\prime} / \rho_{s}\right) \eta(\omega)} .
$$

Here, we have denoted

$$
\begin{aligned}
& \delta N_{\mathrm{td}}^{\omega}=\int_{y<0} d y \sum_{k} \beta_{k}(\omega) \delta Q_{k}^{\omega}, \\
& \eta(\omega)=\int_{y<0} d y \sum_{k} \beta_{k}(\omega) S_{k} .
\end{aligned}
$$

Note that two different processes contribute to the fluctuation of the surface electric field $\delta E_{\perp, s}^{\omega}$. One can see that $\delta N_{\mathrm{td}}^{\omega}$ is related to the random trapping-detrapping processes in the dielectric, while the second integral term in the numerator on the right-hand side of Eq. (29) is due to the random flux of electrons in the channel towards the surface. Both processes are self-consistently coupled by Coulomb correlations between the conducting and trapped electrons.
Having found $\delta E_{\perp, s}^{\omega}$, one can obtain any of the fluctuating quantities given in Eqs. (23)-(25), (28), as well as the spectral density (26) and the excess surface noise [see below, Eq. (43)]. In particular, the spectral correlator $\left\langle\delta E_{\perp, s} \delta E_{\perp, s}^{*}\right\rangle_{\omega}$ by using Eq. (29) reads

$$
\begin{aligned}
\left\langle\delta E_{\perp, s} \delta E_{\perp, s}^{*}\right\rangle_{\omega} & \\
= & \Gamma(\omega)\left\{\left(\frac{q}{\varepsilon}\right)^{2}\left\langle\delta N_{\mathrm{td}} \delta N_{\mathrm{td}}^{*}\right\rangle_{\omega}+\left[\frac{|\eta(\omega)|}{\rho_{s}}\right]^{2}\right. \\
& \times \int_{y>0} d y \rho(y) \int_{y^{\prime}>0} d y^{\prime} \rho\left(y^{\prime}\right) \\
& \left.\times\left\langle\delta r(y) \delta r^{*}\left(y^{\prime}\right)\right\rangle_{\omega}\right\},
\end{aligned}
$$

with

$$
\Gamma(\omega)=\frac{1}{\left|1-\left(\rho_{s}^{\prime} / \rho_{s}\right) \eta(\omega)\right|^{2}} .
$$

For convenience, let $\eta(\omega)=\eta_{1}(\omega)-i \eta_{2}(\omega)$ with positively defined functions $\eta_{1,2}(\omega)$ given by

$$
\eta_{1}(\omega)=\int_{y<0} d y \sum_{k} \frac{S_{k} \tau_{k}}{1+\omega^{2} \tau_{k}^{2}},
$$

and

$$
\eta_{2}(\omega)=\omega \int_{y<0} d y \sum_{k} \frac{S_{k} \tau_{k}^{2}}{1+\omega^{2} \tau_{k}^{2}},
$$

with $\tau_{k}=1 / K_{k}$. By using Eqs. (26) and (29)-(31), we find the expression for the noise power of sheet-electron-density fluctuations

$$
S_{N}(\omega) \equiv\left\langle\delta N \delta N^{*}\right\rangle_{\omega}=M(\omega)[1+G(\omega)] \Gamma(\omega) .
$$

It is considerably modified in comparison with the wellknown result of McWhorter's model. ${ }^{15}$ The factor $M(\omega)$ is due to the conventional McWhorter's mechanism ${ }^{15}$ with disregarded self-consistent electrostatic interactions between the conductive and trapped electrons,

$$
M(\omega) \equiv\left\langle\delta N_{\mathrm{td}} \delta N_{\mathrm{td}}^{*}\right\rangle_{\omega}=\frac{4 n_{s} \eta_{2}(\omega)}{A \omega} .
$$

The second factor in square brackets originates from the transverse electron transport in the channel, with

$$
G(\omega)=\frac{\mathcal{I}_{L} \omega|\eta(\omega)|^{2}}{D \rho_{s}^{2} n_{s} \eta_{2}(\omega)},
$$

and

$$
\mathcal{I}_{L}=\int_{0}^{\infty} n(y)[\rho(y) / W(y)]^{2} d y .
$$

The third factor $\Gamma(\omega)$ on the right-hand side of Eq. (36) has a meaning of the noise-suppression factor, since it is totally due to the Coulomb interactions between electrons incorporated into the model. ${ }^{11}$ It can be shown that $\Gamma(\omega) \leqslant 1$, which follows from its definition given by Eq. (33). The behavior of the latter two factors, extra to McWhorter's formula, will be analyzed in detail in the next section. 


\section{CURRENT-NOISE SPECTRAL DENSITY}

In the previous section, we have obtained the noise power of the carrier-density and electric-field fluctuations in a conducting channel caused by the surface noise. By using these results, we now examine the excess current noise, in particular, the influence of Coulomb correlations on the noise spectrum. We employ the probability distribution function $g(\tau)$ for the decapture time $\tau$, which runs all over the values of the reciprocal rate constants $1 / K_{k}$. The functions $\eta_{1,2}(\omega)$ defined by Eqs. (34) and (35) are expressed in terms of the probability distribution function $g(\tau)$ as

$$
\begin{aligned}
& \eta_{j}(\omega)=\frac{N_{\mathrm{te}}}{n_{s}} Y_{j}(\omega), \\
& Y_{j}(\omega)=\int d \tau(\omega \tau)^{j-1} g(\tau) /\left(1+\omega^{2} \tau^{2}\right),
\end{aligned}
$$

with the index $j=1,2$ and the sheet concentration of trapped electrons $N_{\text {te }}$.

For the mean current along the channel we have

$$
I=q \mu E_{\|} L_{z} \int_{0}^{\infty} n(y) d y .
$$

The noise spectral density of the channel current $I$ is obtained by using Eq. (10) in the form

$$
\begin{aligned}
S_{I}(\omega) & =4 q^{2} N D L_{z} / L_{x}+\left(q \mu E_{\|} L_{z}\right)^{2}\left\langle\delta N \delta N^{*}\right\rangle_{\omega} \\
& \equiv S_{I}^{\mathrm{eq}}+S_{I}^{\mathrm{ex}}(\omega),
\end{aligned}
$$

where $S_{I}^{\mathrm{eq}}=4 k_{B} T / R$ is the equilibrium Johnson-Nyquist contribution, $R=L_{x} /\left(q \mu N L_{z}\right)$ is the channel resistance, and $S_{I}^{\operatorname{ex}}(\omega)$ is the excess surface noise. The latter, under the assumed current-driven operation conditions, may be written as

$$
S_{I}^{\mathrm{ex}}(\omega)=I^{2} \frac{\left\langle\delta N \delta N^{*}\right\rangle_{\omega}}{N^{2}}=I^{2}\left(\frac{\varepsilon}{q N}\right)^{2}\left\langle\delta E_{\perp, s} \delta E_{\perp, s}^{*}\right\rangle_{\omega} .
$$

It is seen that the excess current noise may be equivalently represented either as the number fluctuation noise or the noise of the electric field at the interface. By using the results of the previous section, one gets

$$
S_{I}^{\mathrm{ex}}(\omega)=\Gamma(\omega) S_{I}^{0}(\omega)[1+G(\omega)],
$$

where

$$
S_{I}^{0}(\omega)=\frac{I^{2}}{N^{2}} M(\omega), \quad M(\omega)=\frac{4 N_{\mathrm{te}}}{A} \frac{Y_{2}(\omega)}{\omega},
$$

is the conventional surface-noise term for which the Coulomb correlations are disregarded., ${ }^{4,15}$ The surface-noisesuppression factor $\Gamma(\omega)$ is obtained as

$$
\Gamma(\omega)=\frac{1}{\left[1+\gamma Y_{1}(\omega)\right]^{2}+\gamma^{2} Y_{2}^{2}(\omega)},
$$

with the parameter $\gamma$ defined by

$$
\gamma=\frac{N_{\text {te }}}{n_{s}}\left|\frac{\rho_{s}^{\prime}}{\rho_{s}}\right| .
$$

It is important to note that suppression factor $\Gamma(\omega)$ is a functional of the steady-state solutions taken at the surface $E_{\perp, s}$ and $n_{s}$, which in turn are determined by the surface potential $\varphi_{s}$. Thus, the level of suppression $\Gamma$ in practically important gated structures can be controlled by the gate voltage.

The spectral factor $G(\omega)$ is expressed through the integrals (41) as well:

$$
G(\omega)=\frac{N_{\mathrm{te}} \mathcal{I}_{L}}{D \rho_{s}^{2} n_{s}^{2}} \omega Y_{2}(\omega)\left[1+\frac{Y_{1}^{2}(\omega)}{Y_{2}^{2}(\omega)}\right] .
$$

Thus, as seen from Eqs. (44)-(48), the intensity of the surface noise and its frequency behavior strongly depend on the functions $Y_{1,2}(\omega)$. These functions can be evaluated explicitly by using suitable models for the distribution of traps in the dielectric. Such an analysis will be carried out in Sec. VI, where we consider two different models for distribution of traps in the dielectric: (i) uniformly distributed traps and (ii) traps whose distribution is described by the $\delta$ function.

\section{SPATIAL CORRELATIONS OF NOISE}

The results obtained in the previous sections allow us to study as well the spatial correlations of local fluctuations in the channel and to elucidate the characteristic correlation length $l_{c}$. This is of obvious interest since it gives information about the intensity and frequency dependences of fluctuations taken from different regions of the conduction layer. As an example, we consider the behavior of the local spectral correlator $\left\langle\delta E_{\perp, s} \delta E_{\perp}^{*}(y)\right\rangle_{\omega}$, which gives the magnitude of the correlation between the electric-field fluctuations at the surface and the point located at a distance $y$ inside the channel. By using Eq. (21), it can be expressed as

$$
\left\langle\delta E_{\perp, s} \delta E_{\perp}^{*}(y)\right\rangle_{\omega}=\frac{u_{1}(y)}{u_{1, s}}\left\langle\delta E_{\perp, s} \delta E_{\perp, s}^{*}\right\rangle_{\omega}+\hat{\mathcal{F}}\{h \mid y\},
$$

where $\hat{\mathcal{F}}\{f \mid y\}$ is an integral operator whose kernel depends on $y$ and its action on a function $f(y, \omega)$ is defined according to

$$
\begin{aligned}
\hat{\mathcal{F}}\{f \mid y\}= & u_{1}(y) \int_{0}^{y} u_{2}(\xi) f(\xi, \omega) d \xi \\
& +u_{2}(y) \int_{y}^{\infty} u_{1}(\xi) f(\xi, \omega) d \xi .
\end{aligned}
$$

The spectral correlator $h(y, \omega) \equiv\left\langle\delta E_{\perp, s} \delta r^{*}(y)\right\rangle_{\omega}$ is calculated using Eqs. (29) and (9):

$$
h(y, \omega)=\frac{4 q^{2} \rho(y) n(y)}{\varepsilon^{2} \rho_{s} D A W^{2}(y)} \frac{\eta(\omega)}{1-\left(\rho_{s}^{\prime} / \rho_{s}\right) \eta(\omega)} .
$$

Then, the second term on the right-hand side of Eq. (49) becomes

$$
\frac{4 q^{2}}{\varepsilon^{2} \rho_{s} D A} \hat{\mathcal{F}}\left\{\frac{\rho n}{W^{2}} \mid y\right\} \frac{\eta(\omega)}{1-\left(\rho_{s}^{\prime} / \rho_{s}\right) \eta(\omega)} .
$$


Now, defining the corresponding spectral density for the electric-field fluctuations

$$
S_{E_{\perp}}(y, \omega)=\frac{1}{2}\left[\left\langle\delta E_{\perp, s} \delta E_{\perp}^{*}(y)\right\rangle_{\omega}+\text { c.c. }\right],
$$

we get

$$
\begin{aligned}
S_{E_{\perp}}(y, \omega)= & \frac{q^{2} \rho(y)}{\varepsilon^{2} \rho_{s}} \Gamma(\omega) M(\omega)(1+G(\omega) \\
& \left.+\frac{\omega\left[\eta_{1}(\omega)-\left(\rho_{s}^{\prime} / \rho_{s}\right)|\eta(\omega)|^{2}\right]}{D n_{s} \eta_{2}(\omega)} \frac{1}{\rho} \hat{\mathcal{F}}\left\{\frac{\rho n}{W^{2}} \mid y\right\}\right),
\end{aligned}
$$

with the factor $\hat{\mathcal{F}}\left\{\left(\rho n / W^{2}\right) \mid y\right\}$ determined according to Eq. (50). It is seen that the obtained expression is completely analogous in its structure to Eq. (44) of Sec. IV for the spectral density $S_{I}^{\mathrm{ex}}(\omega)$. It contains the same factors $\Gamma(\omega)$ and $G(\omega)$ originated from the Coulomb correlations and the transverse electron transport, respectively. In Eq. (54), the last factor in parenthesis appears due to the additional cross correlations between the surface and channel electric-field fluctuations coupled by the electron random flux perpendicular to the surface. It is easy to see that Eq. (54) taken at $y$ $=0$ coincides with the surface-noise spectral density obtained previously in Sec. III. Since the spatial dependence of $S_{E_{\perp}}(y, \omega)$ is imposed by the space-charge profile $\rho(y)$ across the nonhomogeneous channel, it is clear that the spatial correlations of fluctuations induced by Coulomb interactions are determined by the Debye screening length $l_{D}$. In other words, the Coulomb correlations in our model result in the frequency-independent correlation length $l_{c}=l_{D}$.

\section{DISCUSSION OF THE RESULTS}

In this section, we apply the results of the developed general theory of surface noise with the incorporated Coulomb correlations for two practically important cases: $1 / f$ noise and the generation-recombination noise spectrum.

\section{A. Traps uniformly distributed over the layer}

As a first example, let us consider the McWhorter model $^{15}$ corresponding to a set of identical traps uniformly distributed in the dielectric, for which the probability distribution function is given by

$$
g(\tau)=\left\{\begin{array}{cc}
C / \tau, & \tau_{1} \leqslant \tau \leqslant \tau_{2}, \\
0, & \text { otherwise. }
\end{array}\right.
$$

Here, the normalization constant $C=\ln ^{-1}\left(\tau_{2} / \tau_{1}\right)$. The difference between the characteristic times $\tau_{1}$ and $\tau_{2}$ may constitute from 5 to 8 decades. ${ }^{15,16}$ These parameters determine the frequency interval

$$
\frac{1}{\tau_{2}}<\omega<\frac{1}{\tau_{1}},
$$

where the surface noise behaves as the flicker $(1 / f)$ noise. The results of our calculations for $\tau_{2} / \tau_{1}=10^{6}$ are presented in Fig. 2. In Fig. 2(a), the relative current noise $S_{I}^{r}(\omega)$
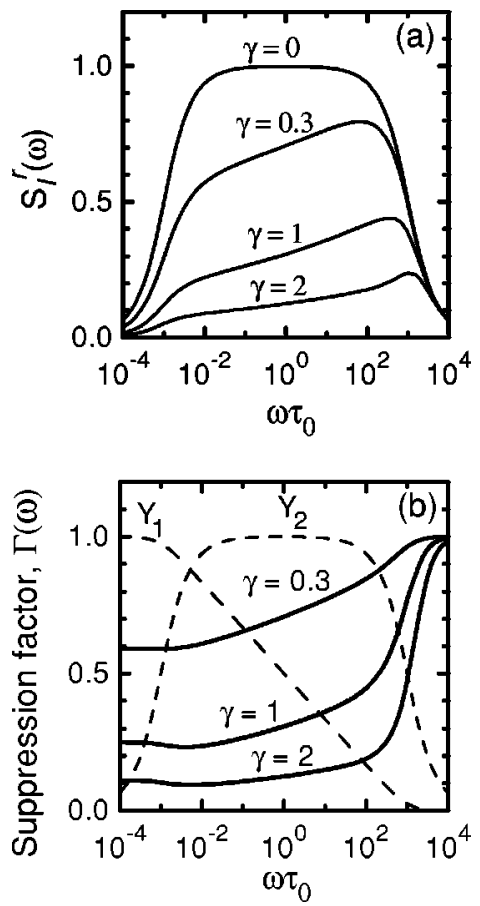

FIG. 2. Frequency dependences of (a) relative current-noise spectral density $S_{I}^{r}(\omega)=S_{I}^{e x}(\omega) / S_{I}^{0}(\omega) \propto \omega S_{I}^{e x}(\omega)$ and (b) suppression factor $\Gamma(\omega)$ (solid) calculated under the trap distribution of the McWhorter model for different values of $\gamma$. Integrals $Y_{1,2}(\omega)$ are also shown (dashes). $Y_{2}(\omega)$ is normalized by its maximum value, $\tau_{0}=\left(\tau_{1} \tau_{2}\right)^{1 / 2}$ and $\tau_{2} / \tau_{1}=10^{6}$.

$=S_{I}^{\mathrm{ex}}(\omega) / S_{I}^{0}(\omega) \propto f S_{I} / I^{2}$, the quantity often used in $1 / f$ noise literature, ${ }^{17}$ is shown. For $g(\tau)$ given by Eq. (55), the integrals (41) become

$$
\begin{aligned}
& Y_{1}(\omega)=C \ln \frac{\tau_{2} \sqrt{1+\omega^{2} \tau_{1}^{2}}}{\tau_{1} \sqrt{1+\omega^{2} \tau_{2}^{2}}}, \\
& Y_{2}(\omega)=C\left[\arctan \left(\omega \tau_{2}\right)-\arctan \left(\omega \tau_{1}\right)\right] .
\end{aligned}
$$

It is easy to verify that $Y_{1}(\omega)$ decays monotonically from 1 at $\omega=0$ to zero at $\omega \rightarrow \infty$. The function $Y_{2}(\omega)$ is nonmonotonic. It increases from $Y_{2}(0)=0$ to its maximum value $Y_{2}^{\max }=C\left(\arctan \sqrt{\tau_{2} / \tau_{1}}-\arctan \sqrt{\tau_{1} / \tau_{2}}\right)$ at $\omega=1 / \tau_{0}$ with $\tau_{0}$ $=\sqrt{\tau_{1} \tau_{2}}$. Then, it decreases to zero as $\omega \rightarrow \infty$. In the frequency range (56), one has approximately $Y_{1}(\omega)$ $\simeq C\left|\ln \left(\omega \tau_{1}\right)\right|$, whereas $Y_{2}(\omega) \simeq C \pi / 2$, i.e., independent of frequency [Fig. 2(b)]. It is important that the function $Y_{2}(\omega)$ has a wide plateau in that frequency range. In the absence of Coulomb correlations, which formally corresponds to $\gamma=0$, this leads to the well-known McWhorter result: $1 / f$ spectrum of the surface noise

$$
S_{I}^{0}(\omega)=I^{2} \frac{N_{\mathrm{te}}}{A N^{2}} \frac{C}{f},
$$

where $f=\omega / 2 \pi$. Outside the frequency interval (56), the spectrum is white at $\omega<1 / \tau_{2}$ and decays as $\omega^{-2}$ at $\omega$ $>1 / \tau_{1}$ [see the curve $\gamma=0$ in Fig. 2(a)].

For the probability distribution (55), the function $G(\omega)$ in Eq. (48) can be approximated at low frequency by $G(0)$ $=G_{0} /\left[C\left(\tau_{2}-\tau_{1}\right)\right] \simeq G_{0} /\left(C \tau_{2}\right)$, and at high frequency it in- 
creases to the value $G(\infty)=C^{2}\left(\tau_{2} / \tau_{1}\right) G_{0}$, where $G_{0}$ $=N_{\mathrm{te}} \mathcal{I}_{L} /\left(D \rho_{s}^{2} n_{s}^{2}\right)$. Finally, within the interval (56), the spectrum of $G(\omega)$ is approximately

$$
G(\omega) \simeq \frac{\pi}{2} G(\infty) \frac{\omega \tau_{1}}{1+\left(4 / \pi^{2}\right) \ln ^{2}\left(\omega \tau_{1}\right)} .
$$

Next, for the suppression factor (46) we obtain at low frequency $\Gamma(0)=1 /(1+\gamma)^{2}$. This is the strongest suppression magnitude over the noise spectrum. It is seen that the level of suppression is determined by the parameter $\gamma$ given by Eq. (47), and it may be significant whenever $\gamma$ is not small with respect to 1 [Fig. 2(b)]. In the high-frequency limit, the suppression effect vanishes, $\Gamma(\infty)=1$. Between these two limits, within interval $(56), \Gamma(\omega)$ is approximated by

$$
\Gamma(\omega) \simeq \frac{1}{\left[1+\gamma C\left|\ln \left(\omega \tau_{1}\right)\right|\right]^{2}+(\gamma \pi C / 2)^{2}} .
$$

One can see that the suppression factor changes only slightly for frequencies $\omega<1 / \tau_{1}$ over many decades. For $\gamma \gtrsim 1$, we obtain $\Gamma(\omega) \approx \Gamma(0)=1 /(1+\gamma)^{2}$, i.e., the suppression factor is approximately constant, as shown by the two lower curves in Fig. 2(b). Thus, we can conclude that when the Coulomb correlations are included into analysis, 1/f law is practically preserved, although with a suppressed value, up to relatively high frequencies. For $\omega>1 / \tau_{1}$, the suppression effect vanishes $[\Gamma(\omega) \approx 1]$, as well as the surface noise, since in this case there are no trap states with characteristic times of the order of $1 / \omega$ and the carrier exchange between the electron channel and the traps becomes ineffective.

It should be noted that Coulomb correlations cause significant changes in the noise spectra. From the above analysis and the results of Fig. 2, it follows that for $\gamma<1$ the shape of $S_{I}^{r}(\omega)$ varies considerably in interval (56). The noise suppression at low frequencies is much more pronounced than at high frequencies. For larger $\gamma$, however, the spectral curves are almost flat, since the suppression factor is nearly constant in this frequency range. It is remarkable that an abrupt increase of $\Gamma(\omega)$ in the vicinity of $\omega \approx 1 / \tau_{1}$, from $\Gamma \approx 1 /(1$ $+\gamma)^{2}$ to $\Gamma \approx 1$ [see Fig. 2(b)], gives rise to a maximum in all the calculated curves of $S_{I}^{r}(\omega)$ in Fig. 2(a). We believe that this characteristic feature of the noise spectrum, when observed in the experiment, could be a valuable test of the effect of Coulomb correlations on the surface noise in semiconductor heterostructures. We would like to emphasize that the shift down of the spectral curves in Fig. 2(a) with increasing $\gamma$ makes evident an absolute noise suppression: the reduction of the noise power at all frequencies, rather than its redistribution over the spectrum. This means that the correspondent integral over the noise spectrum also reduces.

To make numerical estimations, let us consider frequency interval (56) where the surface noise varies as $1 / f$, and its spectral density (44) can be written using the dimensionless Hooge parameter: ${ }^{18,19}$

$$
S_{I}^{\operatorname{ex}}(\omega)=\frac{I^{2}}{f N A} \alpha_{H},
$$

with

$$
\alpha_{H}=\frac{N_{\mathrm{te}}}{N(1+\gamma)^{2} \ln \left(\tau_{2} / \tau_{1}\right)},
$$

being a Hooge parameter ${ }^{19,20}$ modified by the suppression effect. Now, if we take $\gamma=2, N_{\text {te }} / N=0.25$, and $\log \left(\tau_{2} / \tau_{1}\right)$ $=6$, we get $\alpha_{H}=2 \times 10^{-3}$, i.e., the value usually observed in conducting channels with the predominant surface mechanism of the noise. ${ }^{16,18,21}$

Another important consequence of the Coulomb correlations is the prediction of a nonmonotonic dependence of noise spectrum (61) on the concentration of trapped electrons $N_{\text {te }}$. This can be seen by analyzing the Hooge parameter $\alpha_{H}$ as a function of $N_{\text {te }}$. For an electron channel with the local space charge $\rho=-q n$, the parameter $\gamma$ can be expressed explicitly through the electron concentrations $N_{\text {te }}$ and $N$. By using Eqs. (3), (4), and the condition for the transverse steady-state electron flux in the channel $i_{\perp}=0$ [which is analogous to Eq. (17) for fluctuations], we find the following relations: $\rho_{s}^{\prime} / \rho_{s}=-(\mu / D) E_{s}$ and $n_{s}=(\varepsilon \mu / 2 q D) E_{s}^{2}$. Here, the surface field $E_{s}=E_{\perp}(0)$ is related to the electron concentration $N$ through the equation $E_{s}=(q / \varepsilon) N$ [which is analogous to Eq. (25)]. Then, the parameter $\gamma$ is simply $\gamma$ $=2 N_{\text {te }} / N$.

Figure 3 shows the calculated Hooge parameter $\alpha_{H}$ as a function of $N_{\text {te }} / N$. It is seen that without Coulomb correlations, it increases linearly $\alpha_{H} \propto N_{\text {te }} / N$, while for the cases when Coulomb correlations are included, it has a pronounced maximum $\alpha_{H}^{\max }=C / 8$ at $N_{\text {te }} / N=1 / 2$. We also note that for small trap concentrations $N_{\text {te }} \ll N$, the Hooge parameter increases almost linearly for all the cases, since the Coulomb correlations are small to affect noticeably the noise intensity. However, when the concentrations approach $N_{\mathrm{te}} \approx N$, the increase slows down and even changes to the opposite (decreasing) dependence on $N_{\text {te }}$. This effect is entirely due to the Coulomb correlations between the channel carriers and trap states, which for such values of $N_{\text {te }}$ considerably affect the intensity of the surface noise.

Figure 4 shows the spatial correlations of the fluctuations of the transverse electric field across the conducting channel. In Fig. 4, the noise power is normalized according to $S_{E}^{r}(y, \omega)=S_{E_{\perp}}(y, \omega) / S_{E_{\perp, s}}^{0}(\omega) \propto \omega S_{E_{\perp}}(y, \omega)$. Here, $S_{E_{\perp, s}}^{0}(\omega)$ $=(q / \varepsilon)^{2} M(\omega) \propto \omega^{-1}$ corresponds to surface spectral correlator (32) in which the Coulomb correlations and electron transverse transport are ignored. It is seen that the spatial correlations decay on Debye length $l_{D}$ independently of frequency within $1 / f$ noise interval (56) (see, also, the discussion in Sec. V). These results also demonstrate the reduction of spectral intensity of the fluctuations with increasing $\gamma$ caused by Coulomb correlations of charges.

\section{B. $\delta$ distribution of traps}

As another example, we consider the case of a single time constant $\tau_{0}$, describing the exchange of charge carriers between the channel and traps in the dielectric. Unlike uniformly distributed traps, assumed in the McWhorter model, this situation may be relevant to the $\delta$ distribution of traps localized at a certain distance from the interface, as well as to the fluctuations in devices with small areas and low interface 


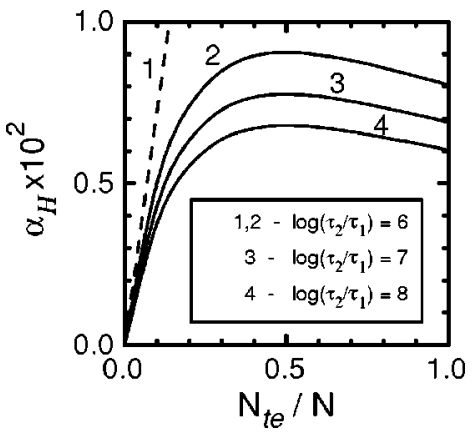

FIG. 3. Illustration of the effect of Coulomb correlations on the Hooge parameter $\alpha_{H}$ plotted vs $N_{t e} / N$ for different $\tau_{2} / \tau_{1}$ ratios: 1 (dashes), $\alpha_{H}$ correspondent to the McWhorter model (no Coulomb correlations); and 2-4 (solid), $\alpha_{H}$ modified by Coulomb correlations.

state densities. ${ }^{20}$ The probability distribution function can be written as $g(\tau)=\delta\left(\tau-\tau_{0}\right)$ with the normalization factor equal to unity. Then, integrals (41) yield

$$
Y_{1}(\omega)=\frac{1}{1+\omega^{2} \tau_{0}^{2}}, \quad Y_{2}(\omega)=\frac{\omega \tau_{0}}{1+\omega^{2} \tau_{0}^{2}} .
$$

The function $Y_{2}(\omega)$ reaches its maximum value $Y_{2}^{\max }=1 / 2$ at $\omega=1 / \tau_{0}$ [Fig. 5(b)]. For these functions, $G(\omega)$ and the suppression factor $\Gamma(\omega)$ read

$$
G(\omega)=\frac{G_{0}}{\tau_{0}}, \quad \Gamma(\omega)=\frac{1+\omega^{2} \tau_{0}^{2}}{(1+\gamma)^{2}+\omega^{2} \tau_{0}^{2}} .
$$

Note that the factor $G$ is independent of frequency. The limiting values for $\Gamma(\omega)$ at $\omega \rightarrow 0$ and $\omega \rightarrow \infty$ are the same as in the previous case. However, in contrast to the McWhorter model with widely distributed trapping/detrapping time parameters, now we get a Lorentzian shape of the excess noise spectrum

$$
S_{I}^{0}(\omega)=I^{2} \frac{4 N_{\mathrm{te}}}{A N^{2}} \frac{\tau_{0}}{1+\omega^{2} \tau_{0}^{2}},
$$

which is modified due to the Coulomb correlations as

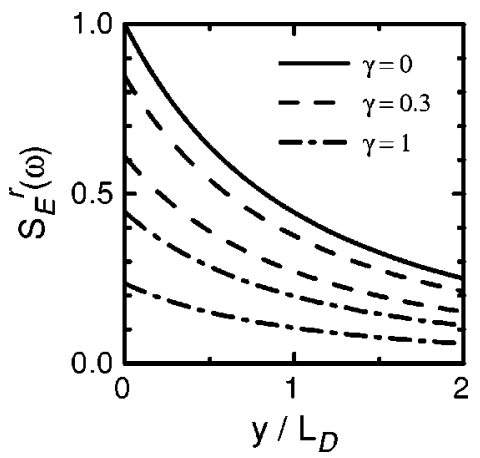

FIG. 4. Illustration of spatial correlations of the local field fluctuations across the channel. Electric-field spectral densities $S_{E}^{r}\left(y, \omega_{i}\right)$ $=S_{E_{\perp}}\left(y, \omega_{i}\right) / S_{E_{\perp}, s}^{0}\left(\omega_{i}\right)$ are plotted for different values of $\gamma$. For $\gamma=0.3$ and $\gamma=1$, each curve is shown for two different frequencies taken from the $1 / f$ interval: $\omega_{1}=0.1 / \tau_{1}$ (upper curve) and $\omega_{2}=10 / \tau_{2}$ (lower curve). $y$ is in units of the Debye length $l_{D}$.
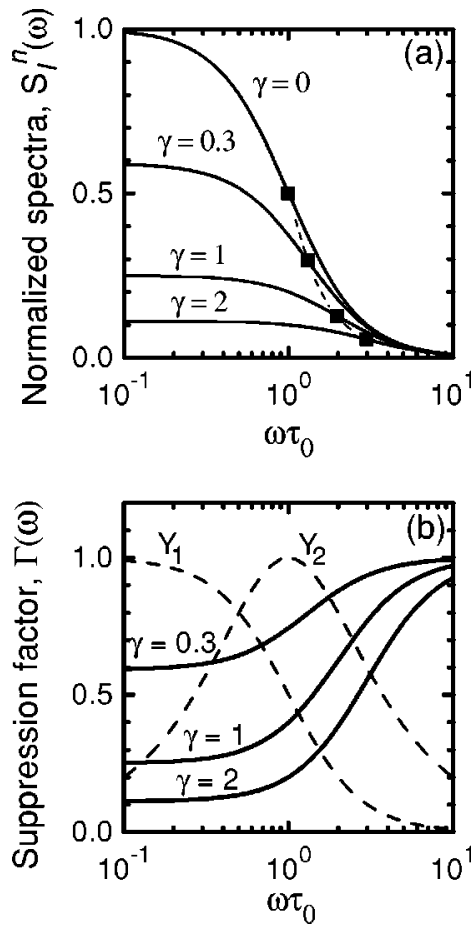

FIG. 5. Frequency dependences of (a) normalized current-noise spectral density $S_{I}^{n}(\omega)=S_{I}^{e x}(\omega) \times\left(A N^{2} / 4 \tau_{0} N_{t e} I^{2}\right)$ and (b) suppression factor $\Gamma(\omega)$ (solid) calculated with the probability distribution function $\delta\left(\tau-\tau_{0}\right)$ for different values of $\gamma$. The integrals $Y_{1,2}(\omega)$ are shown by dashes, where $Y_{2}(\omega)$ is normalized by its maximum value.

$$
S_{I}^{\mathrm{ex}}(\omega)=I^{2} \frac{4 N_{\mathrm{te}}}{A N^{2}} \frac{\tau_{0}}{(1+\gamma)^{2}+\omega^{2} \tau_{0}^{2}} .
$$

Figure 5(a) shows spectra of the normalized currentnoise power $S_{I}^{n}(\omega)=S_{I}^{\mathrm{ex}}(\omega) \times\left(A N^{2} / 4 \tau_{0} N_{\mathrm{te}} I^{2}\right)$ for several values of $\gamma$. The suppression factor $\Gamma(\omega)$ is plotted for the same values of $\gamma$ in Fig. 5(b). The suppression effect due to Coulomb correlations is clearly manifested in several aspects. First, again, the noise power falls down with increasing of $\gamma$, attaining the low-frequency value $S_{I}^{\mathrm{ex}}(0)$ $=S_{I}^{0}(0) /(1+\gamma)^{2}$ at $\omega \tau_{0} \ll 1$. As a result, the total noise power over the whole spectrum $S_{I}^{\mathrm{ex}}$ is also suppressed to $S_{I}^{\mathrm{ex}}=S_{I}^{0} /(1+\gamma)$. Here, $S_{I}^{0}=I^{2}\left(2 \pi N_{\mathrm{te}} / A N^{2}\right)$ is the correspondent total noise power calculated with disregarded Coulomb correlations. Second, since the suppression factor is sensitive to frequency [see Fig. 5(b)], the low-frequency contributions to the noise are much more suppressed than the high-frequency contributions. The noise spectra are broadened with a half-power bandwidth given by $\Delta \omega=(1$ $+\gamma) \Delta \omega_{0}$, where $\Delta \omega_{0}=2 / \tau_{0}$ is the bandwidth with disregarded Coulomb correlations. The corresponding magnitude of the normalized noise power is $S_{I}^{n}\left(\Delta \omega \tau_{0}\right)$ $=1 /\left[2\left(\Delta \omega \tau_{0}\right)^{2}\right]=1 /\left[2(1+\gamma)^{2}\right]$. It is marked by filled squares in Fig. 5(a).

It is convenient to define the dimensionless noise power $S_{I d}^{\mathrm{ex}}(\omega)=S_{I}^{\mathrm{ex}}(\omega) \times\left(A N / \tau_{0} I^{2}\right)$ considered as a function of the ratio $N_{\text {te }} / N$. It has a maximum $S_{I d, m}^{\mathrm{ex}}=1 /\left(1+\sqrt{1+\omega^{2} \tau_{0}^{2}}\right)$ at $N_{\mathrm{te}} / N=(1 / 2) \sqrt{1+\omega^{2} \tau_{0}^{2}}$, with both values dependent on frequency $\omega$. The correspondent dimensionless total noise power can be defined as $S_{I d}^{\mathrm{ex}}=S_{I}^{\mathrm{ex}} \times\left(A N / I^{2}\right)$. The behavior of 


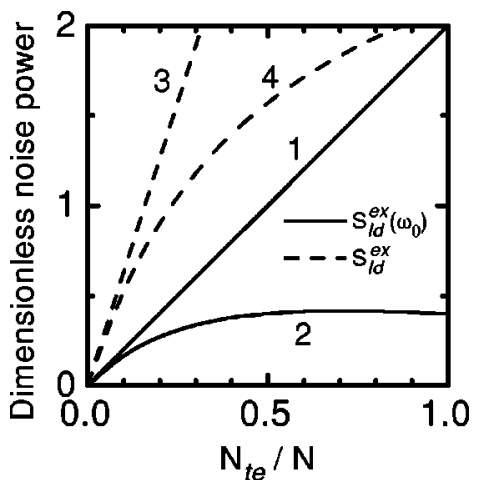

FIG. 6. Dimensionless noise spectral density $S_{I d}^{e x}\left(\omega_{0}\right)$ at $\omega_{0}=1 / \tau_{0}(1,2)$ and total noise power $S_{I d}^{e x}(3,4)$ vs $N_{t e} / N$ calculated with the $\delta\left(\tau-\tau_{0}\right)$ probability distribution function without $(1,3)$ and with $(2,4)$ Coulomb correlations taken into account.

these two quantities is shown in Fig. 6 for two cases: with and without Coulomb correlations taken into account. As seen, both spectral and total noise powers are considerably affected by Coulomb correlations whenever electron densities $N_{\text {te }}$ and $N$ are of the same order of magnitude.

\section{Restrictions of the model}

We conclude this section with the following remarks concerning the validity of the approximations used. In Eq. (15) we assumed that the fluctuations of voltage drop across the dielectric layer due to the change in the self-consistent electrostatic potential do not affect the rate constants. From the comparison of relevant terms on the right-hand side of Eq. (15), one can see that this assumption is justified for $q \delta E_{\perp, s} d /\left(k_{B} T\right) \ll \delta n_{k} / n_{k}$, where $d$ is the thickness of the dielectric layer in which the traps effectively interact with the carriers of the conduction channel. This condition can be rewritten in terms of the sheet electron concentration as $q \delta E_{\perp, s} d /\left(k_{B} T\right) \ll \delta N_{\text {te }} / N_{\text {te }}$. This requires that the relative fluctuation of the concentration of trapped electrons must be much greater than that of the voltage drop across the dielectric layer caused by this fluctuation of $\delta N_{\text {te }}$. Now, by using charge neutrality condition (27), we get $N_{\text {te }} d / N l_{D} \ll 1$. This constraint is fulfilled, since the conditions $d / l_{D} \ll 1$ and $N_{\text {te }} / N \lesssim 1$ usually hold.

One of the assumptions of our model is the short-range character of the potential of traps, which is typical for deep levels in wide-band-gap materials used to design barrier layers in heterostructure samples. If the potential would have a long spatial range, one would need to take into account the screening of the potential. Evidently, for a short-range potential, $r_{0} \ll l_{D}$ ( $r_{0}$ is the effective radius of the trapping potential), the screening effect is not important. Thus, the rate constants in the form of Eqs. (B6) and (B7), given in Appendix B and used in Eq. (15), are justified.

As we pointed out in Sec. I, the change in the selfconsistent electrostatic potential caused by fluctuations of charges on the traps and in the channel is assumed to extend over distances greater than the average distance between the traps. The corresponding condition is expressed by inequality constraint (2). Its right-hand side depends on the parameters
TABLE I. Estimation of characteristic trap concentrations $N_{t 0}$ for electron densities $N$ according to Eq. (2) for conducting channels of different materials at $T=300 \mathrm{~K}$ (the dielectric permittivity $\varepsilon$ is given in parenthesis): $\mathrm{Si}$ (11.8), GaAs (12.8), InAs (12.3), GaN (9.0), and $\mathrm{SiC}$ (9.7). ${ }^{\mathrm{a}}$

\begin{tabular}{cccccc}
\hline \hline$N$ & \multicolumn{5}{c}{$N_{t 0}\left(10^{11} \mathrm{~cm}^{-2}\right)$} \\
\cline { 2 - 6 }$\left(10^{11} \mathrm{~cm}^{-2}\right)$ & $\mathrm{Si}$ & GaAs & InAs & $\mathrm{GaN}$ & $\mathrm{SiC}$ \\
\hline 10 & - & $13^{\mathrm{b}}$ & $2.7^{\mathrm{b}}$ & 190 & 160 \\
5 & 27 & $13^{\mathrm{b}}$ & $2.7^{\mathrm{b}}$ & 47 & 40 \\
1 & 1.1 & 0.95 & 1.0 & 1.9 & 1.6 \\
0.5 & 0.27 & 0.24 & 0.26 & 0.47 & 0.40 \\
\hline \hline
\end{tabular}

${ }^{\text {aSee Ref. } 10}$

${ }^{\mathrm{b}}$ These values were estimated from Eq. (67) corresponding to a quantum screening of charges in the channel and dielectric layer.

of the conducting channel. We list in Table I the characteristic density of traps $N_{t 0}$ estimated for typical conducting channels made of materials used in traditional, as well as advanced, semiconductor heterostructure technologies. ${ }^{10,22,23}$ The results of these estimations indicate that for channels with the electron density $\sim 10^{11} \mathrm{~cm}^{-2}$, the corresponding density of traps $N_{t 0}$ is of the same order of magnitude. For lower electron density in the channel, we obtain $N_{t 0}<N$, while with increasing $N$, the opposite condition $N_{t 0}>N$ holds.

The temperature dependence of the critical trap density $N_{t 0} \propto T^{-2}$ implies another restriction: For a given channel concentration $N$, the higher the temperature, the lower is the critical value of $N_{t 0}$, for which the screening effect of Coulomb correlations in the noise suppression becomes appreciable. On the other hand, at fixed $T$, the increase of $N$ can lead to the regime of quantum screening of charges in the system of two-dimensional conducting channel and dielectric layer. In this regime, Eq. (2) obviously gives overestimated values of $N_{t 0}$, since the Debye screening length becomes independent of the electron density $N: l_{D}=a_{B} / 2$, where $a_{B}$ $=\varepsilon \hbar^{2} / m^{*} q^{2}$ is the Bohr radius and $m^{*}$ is the effective mass. $^{22}$ In the low-temperature limit and for the lowest occupied subband, one can write, similarly to Eq. (2), that

$$
N_{t 0}=\frac{4}{\pi a_{B}^{2}} .
$$

Actually, more rigorous estimations for the self-consistent Coulomb correlations should give a less restrictive condition, since screening in low-dimensional electron systems is known to be less effective. We use formula (67) for calculations of trap density $N_{t 0}$ presented in Table I whenever the quantum screening is relevant. We also note that one of the most important materials to which the developed theory can be applied, is a class of wide-gap nitride-based heterostructures. ${ }^{10}$ The estimates for this material are also reported in Table I. This type of material is known to exhibit considerable surface noise with the Hooge parameter $\gtrsim 10^{-3}$, and they are usually more noisy than the good quality silicon or gallium arsenide-based devices. ${ }^{9}$ 


\section{CONCLUSIONS}

In summary, we have revisited a long-standing problem of the surface noise and proposed a model that includes both capture/decapture of carriers at the surface states and electron transfer towards the surface treated self-consistently. This allowed us to discover a number of features of the surface noise that have not been discussed previously in literature.

We have developed a theory of the surface noise in a nonhomogeneous conducting channel adjacent to an insulating layer. The theory is based on the equations that govern the carrier transport and fluctuations in the electron channel and random trapping/detrapping processes at the channel surface. In contrast to previous studies, it also includes the Coulomb interactions between the trapped and conducting electrons, and inhomogeneous nature of the channel transport. The Coulomb correlations are shown to be essential and should be included into the currently existing simulation tools for device modeling. These correlations suppress the magnitude of the surface noise and could also modify the $1 / f$ form of the noise spectrum in a certain frequency range.

We have studied the spatial correlations of fluctuations and found that the correlation radius is about the Debye screening length. The expression for the Hooge parameter $\alpha_{H}$ for $1 / f$ noise, modified by the presence of Coulomb interactions, has been derived. We have shown that the modified Hooge parameter depends on the concentration of traps, electron density, as well as on the dielectric properties of the structure.

The numerical estimates indicate the significance of the Coulomb correlation effects for the active channels made of silicon, III-V compounds, silicon-carbide, and the group-III nitride-based structures. The studied surface-noisesuppression effect is quite universal and, according to the numerical estimations, may be relevant for a number of practically important devices, like TFTs, BJTs, HFETS, MOSFETs, and, in particular, those used in SOI technology. ${ }^{2}$

\section{ACKNOWLEDGMENTS}

Two of the authors (V.A.K. and V.N.S.) are grateful for partial support by the State Foundation for Fundamental Research of Ukraine and by the Office of Naval Research, Grant No. N00014-01-1-0828. One of the authors (O.M.B.) acknowledges support by the Ministerio de Ciencia y Tecnología of Spain through the "Ramón y Cajal" program.

\section{APPENDIX A: REDUCTION OF BASIC EQUATIONS TO A ONE-DIMENSIONAL FORM}

It will be shown how the system of basic threedimensional (3D) partial differential equations can be reduced to a one-dimensional (1D) form for averaged variables. The original 3D equations governing both the carrier transport and fluctuations are

$$
\begin{aligned}
& \mathbf{i}(\mathbf{r}, t)=-\mu n(\mathbf{r}, t) \mathbf{E}(\mathbf{r}, t)-D \nabla n(\mathbf{r}, t)+\delta \mathbf{J}(\mathbf{r}, t), \\
& \operatorname{div} \mathbf{E}(\mathbf{r}, t)=\frac{\rho(\mathbf{r}, t)}{\varepsilon},
\end{aligned}
$$

$$
\begin{aligned}
& \frac{\partial n(\mathbf{r}, t)}{\partial t}+\operatorname{div} \mathbf{i}(\mathbf{r}, t)=0, \\
& \frac{\partial n_{k}(\mathbf{r}, t)}{\partial t}=S_{k} n(0, t)-K_{k} n_{k}(\mathbf{r}, t)+\delta Q_{k}(\mathbf{r}, t),
\end{aligned}
$$

where $\mathbf{r}=\{x, y, z\}, \mathbf{i}(\mathbf{r}, t)$ is the electron flux, $\delta \mathbf{J}(\mathbf{r}, t)$ the corresponding Langevin noise source, $n(\mathbf{r}, t)$ and $n_{k}(\mathbf{r}, t)$ the electron concentrations in the channel and dielectric, respectively, $\mathbf{E}=-\boldsymbol{\nabla} \varphi$ the electric field, $\varphi(\mathbf{r}, t)$ the electrostatic potential, $\rho(\mathbf{r}, t)$ the channel charge distribution, $\delta Q_{k}(\mathbf{r}, t)$ the trapping noise source, and the rest of notations are analogous to those in Eqs. (3)-(6). These equations must be supplemented by the appropriate boundary conditions.

Next, we apply the double integration over the coordinates $x, z$ in the plane parallel to the interface at a fixed value of the coordinate $y$, and then divide the result by the cross-sectional area $A=L_{x} L_{z}$. Thus, the averaged 1D variables, for example, the electron density $\bar{n}(y, t)$, are introduced as follows:

$$
\bar{n}(y, t)=\frac{1}{A} \int_{0}^{L_{x}} \int_{0}^{L_{z}} n(x, y, z ; t) d x d z .
$$

To illustrate the above approach, consider Poisson equation given by Eq. (A2). Performing the integration and introducing the average variables according to Eq. (A5), we obtain

$$
\begin{gathered}
\frac{\partial \bar{E}_{y}(y)}{\partial y}+\frac{1}{L_{x}}\left\{\bar{E}_{x}\left(L_{x}, y\right)-\bar{E}_{x}(0, y)\right\}+\frac{1}{L_{z}}\left\{\bar{E}_{z}\left(y, L_{z}\right)\right. \\
\left.-\bar{E}_{z}(y, 0)\right\}=\frac{\bar{\rho}(y)}{\varepsilon},
\end{gathered}
$$

where we have denoted

$$
\begin{aligned}
& \bar{E}_{x}(x, y)=\frac{1}{L_{z}} \int_{0}^{L_{z}} E_{x}(x, y, z) d z, \\
& \bar{E}_{z}(y, z)=\frac{1}{L_{x}} \int_{0}^{L_{x}} E_{z}(x, y, z) d x .
\end{aligned}
$$

The derivative in Eq. (A6) can be estimated as

$$
\bar{E}_{y}=-\frac{\partial \bar{\varphi}}{\partial y} \sim-\frac{\bar{\varphi}}{l_{D}}, \quad \Rightarrow \quad \frac{\partial \bar{E}_{y}}{\partial y} \sim-\frac{\bar{\varphi}}{l_{D}^{2}},
$$

where $\bar{\varphi}$ is averaged in two-dimensions according to Eq. (A5). The rest of the terms are of the same order and may be estimated as

$$
\frac{\bar{E}_{x}}{L_{x}} \sim-\frac{\bar{\varphi}}{L_{x} l_{D}}, \quad \frac{\bar{E}_{z}}{L_{z}} \sim-\frac{\bar{\varphi}}{L_{z} l_{D}},
$$

where $\bar{\varphi}$ is averaged in 1D according to Eqs. (A7). It is evident that both averages performed over the electrostatic potential are of the same order of magnitude. Then, for the channels with lateral sizes much greater than the Debye length,

$$
l_{D} \ll L_{x}, L_{z},
$$

the derivative term in Eq. (A6) is dominant, while the next two terms may be omitted, according to Eq. (A9). Thus, the 
$3 \mathrm{D}$ Poisson equation (A2) is reduced to a $1 \mathrm{D}$ form for the averaged (local) field $\bar{E}_{y}$ and the channel space charge $\bar{\rho}$ :

$$
\frac{\partial \bar{E}_{y}(y)}{\partial y}=\frac{\bar{\rho}(y)}{\varepsilon} .
$$

The other 3D equations can be treated in a similar way, by assuming additionally that the conducting channel and the dielectric layer are uniform in the $x$ and $z$ directions.

At this point, we need to perform the averaging in the Langevin sources $\delta J_{\alpha}(\mathbf{r}, t)$ and $\delta Q_{k}(\mathbf{r}, t)$. Their correlation functions have the form ${ }^{4}$

$\left\langle\delta J_{\alpha}(\mathbf{r}, t) \delta J_{\beta}\left(\mathbf{r}^{\prime}, t^{\prime}\right)\right\rangle=2 D_{\alpha \beta} n(\mathbf{r}) \delta_{\alpha \beta} \delta\left(\mathbf{r}-\mathbf{r}^{\prime}\right) \delta\left(t-t^{\prime}\right)$,

$\left\langle\delta Q_{i}(\mathbf{r}, t) \delta Q_{k}\left(\mathbf{r}^{\prime}, t^{\prime}\right)\right\rangle=2 K_{k}(\mathbf{r}) n_{k}(\mathbf{r}) \delta_{i k} \delta\left(\mathbf{r}-\mathbf{r}^{\prime}\right) \delta\left(t-t^{\prime}\right)$,

$\left\langle\delta J_{\alpha}(\mathbf{r}, t) \delta Q_{k}\left(\mathbf{r}^{\prime}, t^{\prime}\right)\right\rangle=0$,

where the angular brackets denote ensemble averages. The coordinate averaging in the above equations leads to the averaged Langevin sources

$$
\begin{aligned}
& \overline{\delta J}_{\alpha}(y, t)=\frac{1}{A} \int_{0}^{L_{x}} \int_{0}^{L_{z}} \delta J_{\alpha}(x, y, z ; t) d x d z, \\
& {\overline{\delta Q_{k}}}_{k}(y, t)=\frac{1}{A} \int_{0}^{L_{x}} \int_{0}^{L_{z}} \delta Q_{k}(x, y, z ; t) d x d z .
\end{aligned}
$$

The correlation functions for these 1D random sources may be evaluated in a standard way ${ }^{4}$ by making use of the correlation functions (A12). Then, we obtain

$$
\begin{aligned}
& \left\langle\overline{\delta J}_{\alpha}(y, t) \overline{\delta J}_{\beta}\left(y^{\prime}, t^{\prime}\right)\right\rangle=\frac{2 D_{\alpha \beta}}{A} n(y) \delta_{\alpha \beta} \delta\left(y-y^{\prime}\right) \delta\left(t-t^{\prime}\right), \\
& \left\langle\overline{\delta Q}_{i}(y, t) \overline{\delta Q}_{k}\left(y^{\prime}, t^{\prime}\right)\right\rangle=\frac{2 K_{k}(y)}{A} n_{k}(y) \delta_{i k} \delta\left(y-y^{\prime}\right) \delta\left(t-t^{\prime}\right),
\end{aligned}
$$

$\left\langle\overline{\delta J}_{\alpha}(y, t) \overline{\delta Q}_{k}\left(y^{\prime}, t^{\prime}\right)\right\rangle=0$.

The Fourier transform of these equations leads to the spectral correlators of the Langevin sources in the form of Eqs. (9) used in the text for the noise analysis (for compactness we omit throughout the text the bar symbol over the averaged variables and random noise sources).

\section{APPENDIX B: RATE CONSTANTS FOR TRAPPING AND DETRAPPING PROCESSES}

Let $N_{S}(E)$ be the effective density of states at the electron energy $E$ in the conduction band of the semiconductor channel, and $N_{D}\left(E_{k}\right)$ be the effective density of states in the dielectric, which depends on discrete trap energy levels $E_{k}$. Introducing the electron thermal velocity normal to the surface $v$ and the trap capture cross section $\sigma_{S D}\left(E, E_{k}\right)$, the probability of capture of an electron with energy $E$ to the trap level $E_{k}$ will be the product $v \sigma_{S D}\left(E, E_{k}\right)$. Then, the elementary electron flux $d i_{k}^{S D}$ from the energy interval between $E$ and $E+d E$ in the semiconductor into the energy interval between $E_{k}$ and $E_{k}+d E_{k}$ of the trap states in the dielectric can be written as

$$
\begin{aligned}
d i_{k}^{S D}= & N_{S}(E) f_{S}(E) v \sigma_{S D}\left(E, E_{k}\right) N_{D}\left(E_{k}\right)[1 \\
& \left.-f_{k}\left(E_{k}\right)\right] d E d E_{k},
\end{aligned}
$$

where $f_{S, k}$ is the Fermi-Dirac distribution function for electrons in the semiconductor $(S)$ and in the localized states $(k)$ of the dielectric.

The net electron flux from the channel to the dielectric layer is obtained by integrating Eq. (B1) over the energies

$$
\begin{aligned}
i_{k}^{S D}= & \int_{E} d E \int_{E_{k}} d E_{k} N_{S}(E) f_{S}(E) v \sigma_{S D}\left(E, E_{k}\right) N_{D}\left(E_{k}\right) \\
& \times\left[1-f_{k}\left(E_{k}\right)\right] .
\end{aligned}
$$

The opposite elementary electron flux from trap states to the channel is written in a similar way

$d i_{k}^{D S}=N_{D}\left(E_{k}\right) f_{k}\left(E_{k}\right) \sigma_{D S}\left(E_{k}, E\right) N_{S}(E)\left[1-f_{S}(E)\right] d E_{k} d E$,

and for the net flux

$$
\begin{aligned}
i_{k}^{D S}= & \int_{E_{k}} d E_{k} \int_{E} d E N_{D}\left(E_{k}\right) f_{k}\left(E_{k}\right) \sigma_{D S}\left(E_{k}, E\right) N_{S}(E) \\
& \times\left[1-f_{S}(E)\right] .
\end{aligned}
$$

The rate constants $S_{k}$ and $K_{k}$ are usually introduced with the relations

$$
i_{k}^{S D}=S_{k} n(0), \quad i_{k}^{D S}=K_{k} n_{k},
$$

and then, according to Eqs. (B2) and (B4), one gets

$$
\begin{aligned}
S_{k}= & \frac{1}{n(0)} \int_{E} d E \int_{E_{k}} d E_{k} N_{S}(E) f_{S}(E) v \sigma_{S D}\left(E, E_{k}\right) \\
& \times N_{D}\left(E_{k}\right)\left[1-f_{k}\left(E_{k}\right)\right],
\end{aligned}
$$

and

$$
\begin{aligned}
K_{k}= & \frac{1}{n_{k}} \int_{E_{k}} d E_{k} \int_{E} d E N_{D}\left(E_{k}\right) f_{k}\left(E_{k}\right) \sigma_{D S}\left(E_{k}, E\right) N_{S}(E) \\
& \times\left[1-f_{S}(E)\right],
\end{aligned}
$$

with the electron concentration at the surface $n(0)$ $=\int_{E} N_{S}(E) f_{S}(E) d E$ and on the traps $n_{k}$ $=\int_{E_{k}} N_{D}\left(E_{k}\right) f_{k}\left(E_{k}\right) d E_{k}$.

In thermal equilibrium, the principle of detailed balance requires that the electron capture and decapture rates should be equal at all points $y$ of the dielectric layer, that is, $v \sigma_{S D}\left(E, E_{k}\right)=\sigma_{D S}\left(E_{k}, E\right)$. As a consequence, both electron fluxes, the direct flux (B2) and the opposite one (B4) are equal as well. Thus, we get a remarkable relation between the rate constants

$$
\frac{S_{k}(y)}{K_{k}(y)}=\frac{n_{k}}{n(0)} .
$$

Now, by using the well-known expressions for the electron concentration at the surface $n(0)=N_{c} \exp \left\{\left[\varepsilon_{F}-E_{c}(0)\right] / k_{B} T\right\}$ and for that on the traps $n_{k}=N_{t k} /\left\{1+(1 / g) \exp \left[\left(E_{k}\right.\right.\right.$ $\left.\left.\left.-\varepsilon_{F}\right) / k_{B} T\right]\right\}$, we find 


$$
\frac{S_{k}(y)}{K_{k}(y)}=\gamma_{k}\left(N_{t k}-n_{k}\right),
$$

where the parameter $\gamma_{k}=\left(g / N_{c}\right) \exp \left[\left(E_{c 0}-E_{k 0}\right) / k_{B} T\right]$ is independent of the Fermi energy $\varepsilon_{F}$. Here, $N_{c}$ is the effective density of states in the conduction band of the semiconductor, $N_{t k}$ is the concentration of traps with energy $E_{k}, g$ is the spin degeneracy factor, $E_{k}(0)=E_{k 0}-q \varphi_{s}$, and $E_{c}(0)=E_{c 0}$ $-q \varphi_{s}$ where the energies $E_{k 0}$ and $E_{c 0}$ correspond to the flatband state $\left(\varphi_{s}=0\right)$.

${ }^{1}$ See, for example, http://www.eetimes.com/news/98/1020news/ ibmtakes.html (October 1998); and more recent http://www.eetimes.com/ story/OEG20010628S0040 (June 2001).

${ }^{2}$ A. Plöß1 and G. Kräuter, Solid-State Electron. 44, 775 (2000).

${ }^{3}$ E. Simoen and C. Claeys, Solid-State Electron. 43, 865 (1999).

${ }^{4}$ Sh. Kogan, Electronic Noise and Fluctuations in Solids (Cambridge University Press, Cambridge, U.K., 1996), Chap. 8

${ }^{5}$ U. H. Liaw and Y. K. Su, J. Appl. Phys. 85, 8485 (1999); J. P. Xu, P. T. Lai, and Y. C. Cheng, ibid. 86, 5203 (1999).

${ }^{6}$ X. Y. Chen, M. J. Deen, A. D. van Rheenen, C. X. Peng, and A. Nathan, J. Appl. Phys. 85, 7952 (1999); S. Giovannini, A. Bove, A. Valletta, L. Mariucci, A. Pecora, and G. Fortunato, Appl. Phys. Lett. 76, 3268 (2000); J. Rhayem, D. Rigaud, M. Valenza, N. Szydlo, and H. Lebrun, J. Appl. Phys. 87, 1983 (2000).

${ }^{7}$ M. J. Deen, S. L. Rumyantsev, and M. Schroter, J. Appl. Phys. 85, 1192 (1999).

${ }^{8}$ P. Sakalas, M. Nawaz, and H. Zirath, Semicond. Sci. Technol. 15, 799 (2000).

${ }^{9}$ S. Rumyantsev, M. E. Levinshtein, R. Gaska, M. S. Shur, J. W. Yang, and M. Asif Khan, J. Appl. Phys. 87, 1849 (2000); S. L. Rumyantsev, N. Pala, M. S. Shur, E. Borovitskaya, A. P. Dmitriev, M. E. Levinshtein, R. Gaska, M. A. Khan, J. Yang, X. Hu, and G. Simin, IEEE Trans. Electron Devices 48, 530 (2001); J. A. Garrido, B. E. Foutz, J. A. Smart, J. R. Shealy, M. J.
Murphy, W. J. Schaff, L. F. Eastman, and E. Muñoz, Appl. Phys. Lett. 76, 3442 (2000).

${ }^{10}$ O. Ambacher, J. Phys. D 31, 2653 (1998); S. J. Pearton, J. C. Zolper, R. J. Shul, and F. Ren, J. Appl. Phys. 86, 1 (1999).

${ }^{11}$ V. A. Kochelap, V. N. Sokolov, O. M. Bulashenko, and J. M. Rubí, Appl. Phys. Lett. 78, 2003 (2001).

${ }^{12}$ S. M. Sze, Physics of Semiconductor Devises, 2nd ed. (Wiley, New York, 1981).

${ }^{13}$ For justification of the Langevin method used here, see, e.g., K. M. van Vliet, J. Math. Phys. 12, 1981 (1971); 12, 1998 (1971); C. M. van Vliet, IEEE Trans. Electron Devices 41, 1902 (1994).

${ }^{14}$ V. A. Kochelap, V. N. Sokolov, and N. A. Zakhleniuk, Phys. Rev. B 48, 2304 (1993); O. M. Bulashenko, G. Gomila, J. M. Rubí, and V. A. Kochelap, J. Appl. Phys. 83, 2610 (1998); V. A. Kochelap and V. N. Sokolov, Phys. Rev. B 57, 15465 (1998); O. M. Bulashenko, J. M. Rubí, and V. A. Kochelap, ibid. 61, 5511 (2000).

${ }^{15}$ A. L. McWhorter, in Semiconductor Surface Physics, edited by R. H. Kingston (University of Pennsylvania Press, Philadelphia, PA, 1957), pp. 207-228.

${ }^{16}$ M. J. Buckingham, Noise in Electronic Devices and Systems (Wiley, New York, 1983), Chap. 6.

${ }^{17}$ L. K. J. Vandamme, X. Li, and D. Rigaud, IEEE Trans. Electron Devices 41, 1936 (1994)

${ }^{18}$ F. N. Hooge, T. G. M. Kleinpenning, and L. K. J. Vandamme, Rep. Prog. Phys. 44, 479 (1981).

${ }^{19}$ A. van der Ziel, Noise in Solid State Devices and Circuits (Wiley, New York, 1986), Chaps. 7.1 and 8.1.

${ }^{20}$ M. Bollu et al., in Proceedings of the Ninth International Conference on Noise in Physical Systems, edited by C. M. Van Vliet (World Scientific, Singapore, 1987), pp. 217-220.

${ }^{21}$ N. Paul, C. M. Van Vliet, and S. Mergui, J. Appl. Phys. 85, 8287 (1999).

${ }^{22}$ V. V. Mitin, V. A. Kochelap, and M. A. Stroscio, Quantum Heterostructures (Cambridge University Press, New York, 1999).

${ }^{23}$ S. Harada, R. Kosugi, J. Senzaki, W. J. Cho, K. Fukuda, K. Arai, and S. Suzuki, J. Appl. Phys. 91, 1568 (2002). 